\title{
Insatisfação corporal e comportamento alimentar inadequado em jovens nadadores segundo níveis econômicos e competitivos
}

\author{
Body dissatisfaction and inappropriate eating behaviors of young \\ swimmers according to economic and competitive status \\ Leonardo de Sousa Fortes', Santiago Tavares Paes', Ana Carolina Soares Amaral', \\ Maria Elisa Caputo Ferreira?
}

\section{RESUMO}

Objetivo: Comparar a insatisfação corporal e o comportamento alimentar entre atletas adolescentes de diferentes níveis econômicos e competitivos. Métodos: Participaram da pesquisa 64 atletas com idade média de 13,64 ( $\pm 2,57)$, sendo 22 do sexo feminino e 42 do masculino. Utilizaram-se o Body Shape Questionnaire (BSQ) e o Eating Attitudes Test (EAT-26) para avaliar insatisfação corporal e comportamento alimentar, respectivamente. A classificação econômica foi obtida mediante aplicação do Critério de Classificação Econômica Brasil (ABEP). Aplicou-se questionário qualitativo para avaliar dados demográficos como idade, sexo e nível competitivo. Resultados: Não se identificaram diferenças de insatisfação nem de comportamento alimentar entre os níveis econômicos e competitivos. Além

\section{Palavras-chave}

Imagem corporal, transtornos alimentares, atletas.

\section{Keywords}

Body image, eating disorders, athletes. disso, os níveis econômicos e competitivos somente modularam a variância do comportamento alimentar $(p<0,05)$. Conclusão: Os resultados do presente estudo permitem concluir que tanto a insatisfação corporal quanto o comportamento alimentar foram semeIhantes entre diferentes níveis econômicos e competitivos em nadadores.

\section{ABSTRACT}

Objective: Compare the eating behavior and body dissatisfaction among adolescent athletes from different economic and competitive levels. Methods: Sixty-four athletes participated in the study with mean age of 13.64 ( \pm 2.57$), 22$ female and 42 male. We used the Body Shape Questionnaire (BSQ) and the Eating Attitudes Test (EAT-26) to assess body dissatisfaction and eating behavior, respectively. The economic classification was obtained by applying the "Brazil Economic Classification Criteria" (ABEP). Qualitative questionnaire was used to evaluate demographic data such as age, gender and competitive level. Results: No differences were identified for dissatisfaction or eating behavior between the economic and competitive levels. In addition, economic and competitive levels only modulated the variance of the eating behavior $(p<0.05)$. Conclusion: The results of this study allow us to conclude that both body dissatisfaction, and eating behavior were similar between different economic and competitive levels of swimmers.

1 Conselho Nacional de Desenvolvimento Científico e Tecnológico (CNPq), Grupo de Pesquisa "Corpo e Diversidade Humana".

Endereço para correspondência: Leonardo de Sousa Fortes Rua Guaçui, 525/202, São Mateus 36025-190 - Juiz de Fora, MG E-mail: leodesousafortes@hotmail.com 


\section{INTRODUÇÃO}

A rotina de treinamento físico intenso e competição à qual atletas estão submetidos parece influenciar o desenvolvimento de pensamentos e atitudes que refletem na saúde física, psicológica e social do indivíduo'. Entre os nadadores, essas características são acompanhadas da exposição frequente do corpo, em função dos trajes competitivos, o que pode influenciar ainda mais os comportamentos em relação ao corpo ${ }^{2}$. Ademais, a pressão exercida por treinadores, clubes e familiares acerca da performance desportiva pode influenciar negativamente a adoção de condutas que esportistas acreditam otimizar o desempenho atlético, como restrição alimentar, indução de vômitos, atividade física extenuante, uso de laxantes e diuréticos ${ }^{3}$. Esse ambiente repleto de cobranças e a tendência estética exigida por algumas modalidades podem fazer com que os atletas desenvolvam preocupações e insatisfações ligadas ao peso e à aparência física ${ }^{4,5}$, repercutindo em sua imagem corporal.

A imagem corporal é um importante construto multidimensional influenciado por fatores psicológicos, neurológicos, culturais e ambientais, que se desenvolve por meio de pensamentos e percepções pessoais sobre as medidas, contornos e estruturas corporais ${ }^{6}$. A imagem corporal pode ser definida como a figura mental que temos acerca de nosso corpo e os sentimentos que possuímos sobre ele'. A insatisfação corporal é um componente afetivo da imagem corporal que envolve aspectos como satisfação com a aparência e o nível de preocupação e ansiedade associado a ela? A relação entre insatisfação corporal, desempenho físico e controle de peso torna os atletas vulneráveis à instalação de comportamentos alimentares inadequados que podem estar associados ao desenvolvimento de transtornos alimentares (TAs).

A anorexia nervosa e a bulimia nervosa são TAs caracterizados por um padrão alimentar gravemente perturbado, um controle patológico do peso corporal, distúrbios da percepção do formato corporal e medo mórbido de engordar ou tornar-se obeso, acompanhados de métodos compensatórios inadequados para o controle da massa corporal, como indução de vômitos, uso indevido de medicamentos (diuréticos, inibidores de apetite, laxantes, anorexígenos), dietas severas e exercícios físicos extenuantes ${ }^{8}$.

Modalidades esportivas que preconizam o baixo peso corporal e enaltecem a estética como um dos critérios de avaliação do desempenho (ginástica artística, ginástica rítmica, nado sincronizado)1,9,10 têm sido apontadas como de grande prevalência de comportamentos considerados precursores de TAs entre seus praticantes, geralmente do sexo feminino ${ }^{11}$. Em contrapartida, pesquisas recentes demonstraram a presença de comportamentos alimentares inadequados também em atletas de modalidades consideradas de baixo risco para TAs (natação, basquete, futebol) 3,12.
Dentre os fatores de risco para TAs, está o nível econômico ${ }^{13}$, pois quanto maior a renda familiar, maior o acesso à informação e aos veículos de mídia, que são componentes indiscutíveis no desenvolvimento dos pensamentos e sentimentos a respeito do corpo. Assim, indivíduos de classes econômicas distintas podem responder e agir de diferentes formas aos estímulos em relação ao corpo que lhes são fornecidos ${ }^{14}$. Estudos têm apresentado resultados controversos a esse respeito. Alguns autores afirmam que os TAs são mais prevalentes nos estratos mais altos da sociedade ${ }^{14}$, enquanto outros estudos não identificaram essa tendência ${ }^{15}$.

Além dos estratos econômicos, o nível competitivo também possui uma forte associação com o aparecimento de alimentação inadequada. Baum ${ }^{16}$ e Denoma et al. ${ }^{4}$ argumentam que atletas de alto nível competitivo podem apresentar maiores riscos para TAs. No entanto, ainda não são encontrados na literatura estudos que tenham avaliado a influência que essas variáveis apresentam sobre a insatisfação corporal e o comportamento alimentar inadequado em atletas competitivos. Portanto, tendo em vista a escassez de estudos que relacionem essas variáveis, o objetivo do presente estudo foi comparar a insatisfação corporal e o comportamento alimentar entre atletas adolescentes de diferentes níveis econômicos e competitivos.

\section{MÉTODOS}

\section{Aspectos éticos}

O estudo obteve aprovação do Comitê de Ética e Pesquisa Envolvendo Seres Humanos da Universidade Federal de Juiz de Fora, com o parecer no 232/2010, de acordo com a Resolução no 196/1996. O Termo de Consentimento Livre e Esclarecido (TCLE), contendo todos os procedimentos da pesquisa, foi assinado pelos pais dos atletas, além de garantir anonimato aos participantes e total sigilo no tratamento dos dados.

\section{População e amostra}

Trata-se de um estudo transversal, cuja população foi constituída por nadadores adolescentes competitivos, de ambos os sexos, com idades de 10 a 19 anos, residentes na cidade de Juiz de Fora/MG. Segundo a Federação Aquática Mineira (FAM), no ano de 2010, essa população era na ordem de 112 atletas. Realizou-se cálculo amostral considerando-se prevalência de 10\% para comportamento alimentar inadequado como efeito de desfecho, com 95\% de confiança, $5 \%$ de erro amostral e efeito de desenho de 1,4, totalizando 63 adolescentes. Para seleção da amostra, foi adotado o critério de amostragem casual simples descrita por Perini et al.'.

A participação foi voluntária e foram excluídos deste estudo os atletas que não apresentaram o TCLE assinado pelos pais, aqueles que não se encontravam em processo de treinamento físico sistematizado com frequência mínima de 
três dias semanais e duração mínima de 1 hora por seção de treino e aqueles que não participaram de pelo menos uma competição regional no ano de 2011.

\section{Instrumentos}

Para avaliar o comportamento alimentar de risco para TAs, foi aplicado o Eating Attitudes Test (EAT-26). Esse é um questionário com 26 questões, em escala Likert de pontos de 0 (nunca) a 3 (sempre), que avalia recusa alimentar patológica, preocupação exacerbada com aparência física, comportamentos purgativos, influência do ambiente na ingestão alimentar e autocontrole sobre os alimentos. Um somatório das respostas igual ou maior que 20 representa indivíduos com comportamento alimentar de risco para TAs. A versão utilizada para o sexo feminino foi validada por Bighetti et al. ${ }^{17}$, apresentando consistência interna de 0,82. Para o sexo masculino, utilizou-se a versão de Fortes et al. (no prelo) ${ }^{18}$, que obteve coeficiente alfa de Cronbach de 0,87. Para a amostra do presente estudo, os valores de alfa de Cronbach foram 0,89 e 0,92 para meninas e meninos, respectivamente, indicando a manutenção das qualidades psicométricas do EAT26 entre os participantes.

Para avaliar a insatisfação corporal, foi aplicado o Body Shape Questionnaire (BSQ). Trata-se de um teste de autopreenchimento com 34 perguntas, em escala Likert de pontos de 1 (nunca) a 6 (sempre), que procura avaliar a preocupação que o sujeito apresenta com seu peso e com sua aparência física. A versão utilizada foi validada para adolescentes brasileiros ${ }^{19}$, obtendo elevada consistência interna $(a=0,96)$ e estabilidade. $\mathrm{O}$ escore é dado pela soma dos itens, e, quanto maior o escore, maior a insatisfação corporal. Segundo o escore, o participante pode ser caracterizado como livre de insatisfação (escores < 80), levemente insatisfeito (entre 80 e 110), moderadamente insatisfeito (entre 110 e 140) e com grave insatisfação corporal (escores > 140). Calculou-se o alfa de Cronbach para a presente amostra, identificando-se valores de 0,89 para as meninas e 0,92 entre os meninos.

A classificação econômica foi obtida mediante aplicação do Critério de Classificação Econômica Brasil, desenvolvido pela Associação Brasileira de Empresas de Pesquisa ${ }^{20}$ (ABEP). O Critério de Classificação Econômica Brasil enfatiza sua função de estimar o poder de compra das pessoas e famílias urbanas, abandonando a pretensão de classificar a população em termos de "classes sociais". Esse instrumento avalia a quantidade de itens de conforto (automóvel, geladeira, televisão etc.) adquiridos, além de identificar o grau de instrução do chefe de família. Ele nos remete aos seguintes pontos de corte em relação à classe econômica: A1 - 30 a 34 pontos; A2 - 25 a 29; B1 - 21 a 24; B2 - 17 a 20; C - 11 a 16; D - 6 a 10; E - 0 a 5, em ordem decrescente de nível econômico.

Foi aplicado um questionário qualitativo a fim de se avaliarem dados demográficos como: idade, sexo, nível competitivo (regional, estadual ou nacional) e horas de treino por dia.

\section{Procedimentos}

O estudo foi realizado no local de treinamento, após autorização dos treinadores e devolução do TCLE assinado pelos responsáveis. Os questionários (EAT-26 e BSQ) foram aplicados individualmente, por um mesmo pesquisador (LSF), e não houve limite de tempo para as respostas.

\section{Análise dos dados}

Foram realizadas análises descritivas (média e desvio-padrão) das variáveis "insatisfação corporal" e "comportamento alimentar". Considerou-se que as variáveis do estudo eram do tipo "não paramétrica", por se tratarem de dados comportamentais e afetivos que utilizam escalas do tipo likert 5 . O teste Kruskal-Wallis foi utilizado para comparar os escores de "insatisfação corporal" e "comportamento alimentar" entre diferentes níveis econômicos e competitivos. Para identificar tais diferenças, utilizou-se o post hoc de Bonferroni. Para isso, as classificações do ABEP foram agrupadas em A (A1, $A 2), B(B 1$ e B2) e C (C, D e E). Foi conduzida análise de regressão múltipla stepwise para identificar a influência exercida pelos níveis econômicos e competitivos sobre a "insatisfação corporal" e o "comportamento alimentar". Todos os dados foram tratados no software SPSS versão 17.0, com nível de significância de 5\%.

\section{RESULTADOS}

Participaram da pesquisa 64 atletas com idade média de 13,64 ( $\pm 2,57)$, sendo 22 do sexo feminino e 42 do masculino. A distribuição da amostra segundo nível econômico e competitivo está apresentada na tabela 1.

Tabela 1. Distribuição da frequência de nadadores segundo níveis econômicos e competitivos

\begin{tabular}{lcc}
\hline Variável & N & Frequência (\%) \\
\hline Nível econômico (ABEP) & & \\
A & 13 & 20,3 \\
B & 45 & 70,3 \\
C & 6 & 9,4 \\
Nível competitivo & & \\
Regional & 16 & 25 \\
Estadual & 42 & 65,6 \\
Nacional & 6 & 9,4 \\
\hline
\end{tabular}

Em relação à insatisfação corporal entre participantes dos diferentes níveis econômicos, não foi verificada diferença significativa, como apresentado na tabela 2. Entretanto, os valores permitem inferir que quanto menor o estrato econômico, maior a insatisfação corporal avaliada pelo BSQ.

Remetendo-se aos valores obtidos para o EAT-26 em cada estrato econômico, não foram observadas diferenças 
significativas entre os escores nos três estratos. Apesar disso, observou-se uma tendência de que quanto menor o estrato social, maior o escore obtido no EAT-26 (Tabela 2).

A respeito do nível competitivo, não foram encontradas diferenças significativas em relação às variáveis insatisfação corporal e comportamento alimentar, como pode ser observado na tabela 3.

A análise de regressão múltipla demonstrou que somente o nível econômico exerceu influência sobre a insatisfação corporal $(9 \%)(p<0,05)$. No entanto, parece que tanto o nível econômico (11\%) quanto o competitivo (7\%) modularam a variância do comportamento alimentar. Além disso, a interação entre esses níveis (econômico e competitivo) explicou em 13\% a variância da pontuação do EAT-26 $(p<0,05)$.

Tabela 2. Média e desvio-padrão da insatisfação corporal e do comportamento alimentar de nadadores adolescentes competitivos em diferentes classificações econômicas

\begin{tabular}{lcccc}
\hline ABEP & A & B & C & p valor \\
\hline BSQ & $50,54 \pm 14,11$ & $67,93 \pm 25,48$ & $68,83 \pm 37,91$ & 0,055 \\
EAT-26 & $6,62 \pm 5,36$ & $9,91 \pm 7,99$ & $12,00 \pm 9,20$ & 0,079 \\
\hline
\end{tabular}

Tabela 3. Média e desvio-padrão da insatisfação corporal e do comportamento alimentar de nadadores adolescentes competitivos em diferentes níveis competitivos

\begin{tabular}{lcccc}
\hline \multicolumn{1}{c}{ NC } & Regional & Estadual & Nacional & pvalor \\
\hline BSQ & $66,31 \pm 33,97$ & $66,24 \pm 25,65$ & $57,33 \pm 19,75$ & 0,630 \\
EAT-26 & $10,00 \pm 8,03$ & $9,40 \pm 8,22$ & $5,50 \pm 2,73$ & 0,107 \\
\hline NC: nível competitivo. & & &
\end{tabular}

\section{DISCUSSÃO}

O presente estudo buscou comparar a insatisfação corporal e o comportamento alimentar entre atletas adolescentes de diferentes níveis econômicos e competitivos. Os resultados deste estudo não evidenciaram diferenças estatisticamente significativas de insatisfação corporal entre os diferentes níveis econômicos. No entanto, pesquisas têm demonstrado que a insatisfação corporal aflige com maior gravidade sujeitos inseridos em níveis econômicos mais altos ${ }^{14,15}$. Essa diferença pode ser justificada por causa da diferenciação da amostra, considerando que a maior parte dos estudos que demonstraram o aumento da insatisfação em relação à classe econômica foi realizada em escolares.

O nível econômico respondeu por somente 9\% da variância da insatisfação corporal. Por outro lado, o nível competitivo não exerceu influência sobre essa variável afetiva. Parece que cerca de $90 \%$ da modulação da insatisfação corporal de nadadores de Juiz de Fora podem ser explicados por outros fatores. Alguns autores destacam a importância dos fatores sociais no desenvolvimento de insatisfação corporal, ressaltando o papel dos pais, amigos e mídia sobre esse constru- to ${ }^{21}$. Talvez pressões de treinadores cobrando a otimização do rendimento físico e o uso de vestimentas que expõem o corpo possam ser tópicos que também expliquem a variância desse fenômeno nesta amostra².

Remetendo-se ao nível competitivo, não se encontraram diferenças significativas de insatisfação corporal entre atletas que competiam em âmbito regional, estadual e nacional. Entretanto, alguns autores ${ }^{1,4}$ sugerem que altos níveis competitivos podem provocar maiores preocupações e depreciações com peso e aparência corporal.

Em relação ao comportamento alimentar, não foram encontradas diferenças de pontuações do EAT-26 entre as classificações econômicas. Em contrapartida, outros estudos revelaram que quanto mais alto o nível econômico, maior a ocorrência de comportamentos alimentares de risco ${ }^{14,15}$.

A respeito das comparações de comportamento alimentar entre os níveis competitivos, mais uma vez não se encontraram diferenças significativas. Entretanto, estudos como de Denoma et al. ${ }^{4}$ demonstraram resultados diferentes, indicando que quanto mais elevado o nível competitivo, maior a prevalência de comportamentos alimentares inadequados.

Remetendo-se à influência de níveis econômicos e competitivos sobre o comportamento alimentar, alguns resultados merecem destaque. Tanto o nível econômico quanto o competitivo modularam as pontuações do EAT-26. Além disso, a interação entre esses níveis explicou cerca de 13\% da variância do comportamento alimentar. De qualquer forma, parece que hábitos alimentares de atletas são comportamentos de extrema complexidade, pois aproximadamente 87\% desse fenômeno são explicados por outros fatores, permanecendo, então, como tema a ser mais bem explorado no campo acadêmico 3 .

O presente estudo apresentou limitações. Pesquisadores afirmam que os participantes podem não responder a questionários autoaplicáveis com fidedignidade, ainda mais tratando-se de atletas, que podem associar sua participação à permanência na equipe. Portanto, é comum que atletas, mesmo com problemas psicológicos, mascarem suas síndromes omitindo-as por meio de respostas não confiáveis a partir de instrumentos desse tipo. Além disso, variáveis como índice de massa corporal e gordura relativa, que, de alguma forma, podem influenciar na insatisfação corporal e no comportamento alimentar, não foram mensurados a fim de serem utilizadas como covariáveis no tratamento dos dados do presente estudo.

Entretanto, este estudo apresentou resultados importantes que podem indicar que atletas adolescentes competitivos de natação podem responder de forma diferente à exigência em relação ao corpo e à alimentação. São necessários estudos que busquem comparar as variáveis aqui analisadas em outras modalidades esportivas, a fim de investigar o comportamento delas em outros âmbitos esportivos. 


\section{CONCLUSÃO}

Os resultados do presente estudo permitem concluir que tanto a insatisfação corporal quanto o comportamento alimentar foram semelhantes entre diferentes níveis econômicos e competitivos de nadadores de Juiz de Fora/MG. Ademais, os resultados permitem inferir que os níveis econômicos e competitivos mediaram as variações no comportamento alimentar, mas não na imagem corporal, sobre a qual apenas o nível econômico demonstrou influência estatística.

\section{AGRADECIMENTOS}

Aos atletas participantes deste estudo, pela colaboração. Ao Laboratório de Estudos do Corpo (UFJF), pela disponibilização dos instrumentais do estudo. Ao Conselho Nacional de Desenvolvimento Cientííco e Tecnológico (CNPq), pelo financiamento do projeto.

\section{REFERÊNCIAS}

1. Perini TA, Vieira RS, Vigário OS, Oliveira GL, Ornellas JS, Oliveira FP. Transtorno do comportamento alimentar em atletas de elite de nado sincronizado. Rev Bras Med Esporte. 2009;15(1):54-7.

2. Swami V, Steadman L, Toveé M. A comparison of body size ideals, body dissatisfaction, and media influence between female track athletes, martial artists, and non-athletes. Psychol Sport Exerc. 2009;10(1):609-14.

3. Schaal K, Tafflet M, Nassif H, Thibault V, Pichard C, Alcotte M, et al. Psychological balance in high level athletes: gender-based differences and sport-specific patterns. PLOS One. 2011;6(5):e19007.

4. Denoma JMH, Scaringi V, Gordon KH, Van Orden KA, Joiner TE. Eating disorder symptoms among undergraduate varsity athletes: club athletes, independent exercisers and nonoexercises. Int J Eat Disord. 2009;12(1):47-53.

5. Sundgot-Borgen J, Torstveit MK. Prevalence of disorders in elite athletes in higher than in the general population. Clin J Sport Med. 2004;14(1):25-32.
6. Fidelix YL, Silva DAS, Pelegrini A, Silva AF, Petroski EL. Insatisfação com a imagem corporal em adolescentes de uma cidade de pequeno porte: associação com sexo, idade e zona de domicílio. Rev Bras Cineantropom Desempenho Hum. 2011;13(3):202-7.

7. De Bruin AP, Oudejans RRD, Bakker FC. Dieting and body image in aesthetic sports: a comparison of dutch female gymnnasts and non-aesthetic sport participants. Psychol Sport Exerc. 2007:8(4):507-20.

8. Phillipp ST, Alvarenga M. Transtornos alimentares: uma visão nutricional. 2a ed. São Paulo; Manole; 2004.

9. Oliveira FP, Bosi MLM, Vigario OS, Viveira RS. Comportamento alimentar e imagem corporal em atletas. Rev Bras Med Esporte. 2003;9(6):348-56.

10. Vieira $J L L, A m o r i m ~ H Z$, Vieira LF, Amorim AC, Rocha PGM. Distúrbios de atitudes alimentares e distorção da imagem corporal no contexto competitivo da ginástica rítmica. Rev Bras Med Esporte. 2009;15(6):410-4.

11. Assunção SSM, Cordás TA, Araújo LASB. Atividade física e transtornos alimentares. Rev Psiq Clín. 2002;29(1):4-13.

12. Fortes LS, Ferreira MEC. Comparação da insatisfação corporal e do comportamento alimentar inadequado em atletas adolescentes de diferentes modalidades esportivas. Rev Bras Educ Fís Esporte. 2011;25(4):707-16.

13. Dunker KLL, Fernandes CPB, Carreira Filho D. Influência do nível socioeconômico sobre comportamentos de risco para transtornos alimentares em adolescentes. J Bras Psiquiatr. 2009;58(3):156-61.

14. Vale AMO, Kerr LRS, Bosi MLM. Comportamento de risco para transtornos do comportamento alimentar entre adolescentes do sexo feminino de diferentes estratos sociais do Nordeste do Brasil. Ciênc Saúde Coletiva. 2011;16(1):121-32.

15. Pereira EF, Graup S, Lopes AS, Borgatto AF, Daronco LSE. Percepção da imagem corporal de crianças e adolescentes com diferentes níveis socioeconômicos na cidade de Florianópolis, Santa Catarina, Brasil. Rev Bras Saúde Mater Infantil. 2009;9(3):253-62.

16. Baum A. Eating disorders in the male athlete. Sports Med. 2006;36(1):1-6.

17. Bighetti F, Santos CB, Santos JE, Ribeiro RPP. Tradução e avaliação do Eating Attitudes Test em adolescentes do sexo feminino de Ribeirão Preto, São Paulo. J Bras Psiquatr. 2004;53(6):339-46.

18. Fortes LS, Amaral ACS, Conti MA, Cordás TA, Ferreira MEC. Qualidades psicométricas do Eating Attitudes Test (EAT-26) para a população adolescente masculina brasileira. Psicologia: Reflexão e Crítica (no prelo).

19. Conti MA, Cordás TA, Latorre MRDO. Estudo de validade e confiabilidade da versão brasileira do Body Shape Questionnaire (BSQ) para adolescentes. Rev Bras Saúde Mater Infantil. 2009;9(3):331-8.

20. Critério de Classificação Econômica Brasil. Disponível em: http://www.datavale-sp.com. br/CCEB.pdf. Acessado em: Fev 1, 2011.

21. Cafri G, Thompson JK, Ricciardelli L, McCabe M, Smolak L, Yesalis C. Pursuit of the muscular ideal: physical and psychological consequences and putative risk factors. Clin Psychol Rev. 2005;25(2):215-39. 